\title{
Transition temperature(s) of magnetized two-flavor holographic QCD
}

\author{
N. Callebaut* and D. Dudal ${ }^{\dagger}$ \\ Ghent University, Department of Physics and Astronomy, Krijgslaan 281-S9, 9000 Gent, Belgium
}

(Received 25 March 2013; published 3 May 2013)

\begin{abstract}
During heavy ion collisions, high temperatures and strong magnetic fields are generated. We employ the gauge-gravity duality to study the $N_{f}=2$ QCD phase diagram under these extreme conditions in the quenched approximation; in particular we use the non-antipodal Sakai-Sugimoto model (SSM). We take the different coupling of up and down flavors to the magnetic field into account geometrically, resulting in a split of the chiral phase transition according to flavor. We discuss the influence of the magnetic field on the chiral temperatures - in physical GeV units - in terms of the choice of the confinement scale in the model, extending hereby our elsewhere presented discussion of fixing the non-antipodal SSM parameters to the deconfinement phase. The flavor-dependent $(T, L, e B)$ phase diagram, with variable asymptotic brane-antibrane separation $L$, is also presented, as a direct generalization of the known $(T, L)$ phase diagram of the non-antipodal SSM at zero magnetic field. In particular, for sufficiently small $L$ we are probing a Nambu-Jona-Lasinio (NJL)-like boundary field theory in which case we do find results very reminiscent of the predictions in NJL models.
\end{abstract}

DOI: $10.1103 /$ PhysRevD.87.106002

PACS numbers: 11.25.Tq, 12.38.Aw

\section{MOTIVATION}

It has already been known for some time that gigantic magnetic fields occurred during the cosmological electroweak phase transition [1], but recently it has also become clear that magnetic fields up to $10^{15} \mathrm{~T}$ can occur shortly during relativistic heavy ion collisions; see e.g. [2,3]. To put such a field strength in the correct perspective, a magnetar, or highly magnetized neutron star, reaches "merely" $10^{9} \mathrm{~T}$. The interest in QCD studies under these extreme conditions has therefore increased considerably. As strong coupling effects are relevant in the setting of interest, we must rely on nonperturbative tools to study the relatively new realm of QCD physics in a strong magnetic background. This has initiated a vast amount of original research; let us refer to [4] and references therein for a recent review. In particular, confinement and chiral symmetry breaking are two typical nonperturbative QCD effects that can be affected. It is common knowledge that QCD deconfines at a certain temperature $T_{c}$, while chiral symmetry is restored at $T_{\chi}$. We shall be concerned with QCD in the chiral limit here, i.e. we ignore the bare quark masses, in which case a clear-cut chiral transition exists. In real life QCD, with massive dynamical flavors, only approximate order parameters can be defined for both chiral restoration ( $\sim$ chiral condensates) and deconfinement ( $\sim$ Polyakov loop), leading to crossover behavior rather than sharp phase transitions. Once a hot debate whether $T_{c}$ and $T_{\chi}$ coincided (see [5,6] for two views on the $N_{f}=$ $2+1$ case), it is by now accepted that they are close in the crossover region. These results were obtained using lattice

\footnotetext{
*ncalleba.callebaut@ugent.be

†david.dudal@ugent.be
}

simulations, a powerful ally to access the nonperturbative QCD sector.

More recently, a vivid discussion evolved around the possibility that $T_{c}$ and $T_{\chi}$ separate under the influence of a constant magnetic background field, $\mathbf{B}=B \mathbf{e}_{z}$, and this for $N_{f}=2$ QCD; see e.g. [7-9]. Although the $N_{f}=2$ lattice results of [9] indicated a weak rise in the transition temperatures $T_{c}$ and $T_{\chi}$, both remained compatible with each other (a split of $\sim 2 \%$ ), while the various analytical model based results were inconclusive on the matter, as different results were obtained per QCD model $[7,8]$. Somewhat later, a more thorough lattice study appeared using $N_{f}=$ $2+1$ flavors with physical masses, leading to a much more complicated behavior in the chiral/deconfinement (pseudo-)order parameters and ensuing critical temperatures [10]. It was motivated that the reported behaviorwhere contrasting with the results of [9]—should be traced back to the lighter dynamical flavors and partially also to the present strange flavor, as the up $(u)$ and down $(d)$ quarks of [9] were considerably heavier. Soon after, the first analytical papers appeared trying to explain the stateof-the-art lattice data using backreacting pion dynamics [11]. The naive reason for expecting a split between $T_{c}$ and $T_{\chi}$ was the expected enhancement of chiral symmetry breaking due to a magnetic field - the so-called chiral magnetic catalysis [12]. The results of [10] showed a more subtle picture: the magnetic catalysis was confirmed for temperatures (sufficiently) below $T_{c}$, but for larger temperatures the (averaged over up and down) chiral condensate displayed a nonmonotonous shape, a feature translated into a similar behavior in the transition temperature. This observation of an "inverse magnetic catalysis" seems to depend crucially on taking into account quark backreaction effects (see also [13]), so we do not expect it to appear in the unquenched Sakai-Sugimoto setting we will use. 
Since a magnetic field couples to the up and down flavors with another strength, as they carry different electric charges, it seems natural that the up and down chiral restoration temperatures can be different, as well as the magnetic catalysis itself. We recall that the classical chiral structure of QCD with and without magnetic field is different, since coupling a magnetic field to the quarks reduces $U(2)_{L} \times U(2)_{R}$ to $\left[U(1)_{L} \times U(1)_{R}\right]^{u} \times\left[U(1)_{L} \times U(1)_{R}\right]^{d}$, so that the eventually broken chiral invariances $U(1)_{A}^{u}$ and $U(1)_{A}^{d}$ can experience a different restoration temperature. Lattice simulations indeed confirm a larger value for the $\langle\bar{u} u\rangle$ than for the $\langle\bar{d} d\rangle$ chiral condensate at $T=0$ [14], as does the $N_{f}=2$ Nambu-Jona-Lasinio (NJL) model [15]. It would appear natural that $T_{\chi}^{u}$ should consequently be larger than $T_{\chi}^{d}$. The splitting of degenerate order parameters, like $\langle\bar{u} u\rangle$ and $\langle\bar{d} d\rangle$ at $e B=0$, when an external field is switched on, is not that unfamiliar. In certain exotic superconductors, e.g. $\mathrm{Sr}_{2} \mathrm{RuO}_{4}$, a similar phenomenon occurs [16].

Here, we will use the gauge-gravity duality, a powerful tool to analytically study certain aspects of strongly coupled gauge theories [17], to shed further light on the possible $(T, e B)$ QCD phase diagram. In particular, we rely on the non-antipodal Sakai-Sugimoto model (SSM) [18] with two quenched flavors, represented by two D8- $\overline{\mathrm{D} 8}$ probe brane-antibrane pairs, in the chiral limit and with three colors. Going beyond the quenched approximation and/or including massive dynamical flavors leads to utter complications $[19,20]$. We take into due account the different coupling of up and down flavors to the magnetic field, leading to a different probe brane geometry per flavor. We compare our findings with the lattice results of [21]. Although those results are referring to two-color QCD, it contains an extrapolation to the chiral limit, which is the closest the available lattice results come to the $N_{f}=2$ SSM. ${ }^{1}$ In [21], no manifest split between $T_{c}$ and $T_{\chi}$ was reported, while the chiral condensate increases monotonically with the applied magnetic field for all temperatures in the confinement phase. We do present similar results here using prefixed values for the string theory parameters of the SSM. With prefixed, we mean that a few physical QCD input values at zero magnetic field are chosen to match the corresponding SSM predictions. These results are a generalization to the two flavor case of the single flavor analysis of [22]. To extend the scope of our analysis, we will also allow that the asymptotic D8- $\overline{\mathrm{D} 8}$ separation $L$-or equivalently, the confinement scale $M$-can vary, and as such we construct the magnetic generalization of the $(T, L)$ phase diagram of the SSM, displayed in Fig. 7 of the original work [23]. All results are presented in $\mathrm{GeV}$

\footnotetext{
${ }^{1}$ We will use $N_{c}=3$ colors to get explicit numbers, where in principle the limit $N_{c} \rightarrow \infty$ is always understood at the holographic level. Setting $N_{c}=2$ or $N_{c}=3$ will not induce any qualitative change in the SSM results.
}

units to make comparison with other QCD approaches more direct.

\section{SETUP}

\section{A. The Sakai-Sugimoto model}

At zero temperature, the SSM [18] involves a system of $N_{f}$ pairs of D8- $\overline{\mathrm{D} 8}$ flavor probe branes placed in the D4-brane background

$$
\begin{aligned}
d s^{2}= & \left(\frac{u}{R}\right)^{3 / 2}\left(\eta_{\mu \nu} d x^{\mu} d x^{\nu}+f(u) d \tau^{2}\right) \\
& +\left(\frac{R}{u}\right)^{3 / 2}\left(\frac{d u^{2}}{f(u)}+u^{2} d \Omega_{4}^{2}\right), \\
f(u)= & 1-\frac{u_{K}^{3}}{u^{3}}
\end{aligned}
$$

where $R^{3}=\pi g_{s} N_{c} \ell_{s}^{3}$, with $g_{s}\left(\ell_{s}\right)$ the string constant (length). There is a natural cutoff at $u=u_{K}$, which ensures confinement in the dual field theory living at the boundary $u \rightarrow \infty$. A smooth cutoff is realized if $\tau$ has a periodicity $\delta \tau=\frac{4 \pi}{3} R^{3 / 2} u_{K}^{-1 / 2}=2 \pi M^{-1}$ with $M$ the compactification scale. A $U\left(N_{f}\right)_{L} \times U\left(N_{f}\right)_{R}$ gauge theory resides on the stack of coinciding D8-D8 flavor pairs, which corresponds to the global chiral symmetry in the dual QCD-like theory. The cigar shape of the $(u, \tau)$ subspace of the D4-brane background forces the embedding of the flavor branes to be $\cup$ shaped, which signals the breaking of chiral symmetry $U\left(N_{f}\right)_{L} \times U\left(N_{f}\right)_{R} \rightarrow U\left(N_{f}\right)_{V}$ as the merging of the D8-branes and $\overline{\mathrm{D} 8}$-branes at the value $u=u_{0} \geq u_{K}$. The value $u_{0}$ is directly related to the asymptotic separation $L$ (at $u \rightarrow \infty$ ) between D8-branes and $\overline{\mathrm{D} 8}$-branes, indicated in Fig. 1. For values of $u_{0}$ greater than or equal to $u_{K}$ the embedding is, respectively, non-antipodal or antipodal.

At finite temperature there are two regular Euclidean backgrounds with the same asymptotic geometry that compete with each other in the partition function, the Wick-rotated version of the D4-brane background (1) and

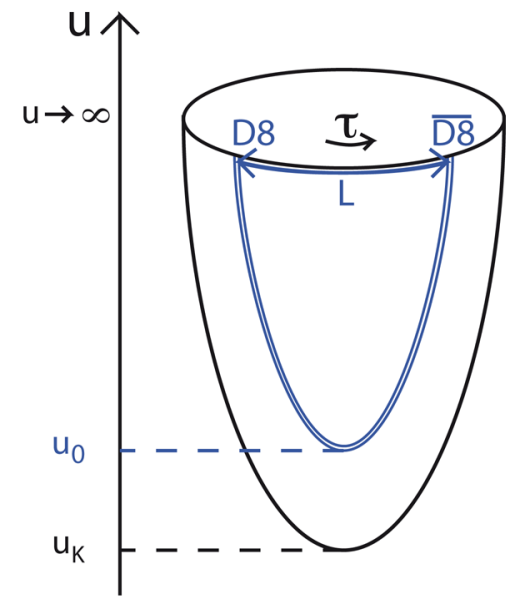

FIG. 1 (color online). The Sakai-Sugimoto model. 
a black D4-brane background [23]. The Wick-rotated D4-brane background has a cigar-shaped $(u, \tau)$ subspace and a cylinder-shaped $(u, t)$ subspace, with the periodicity of the $t$ circle arbitrary and equal to the inverse temperature, $\beta=T^{-1}$, and the periodicity of the $\tau$ circle fixed to $\delta \tau=\frac{4 \pi}{3} R^{3 / 2} u_{K}^{-1 / 2}$, while the black D4-brane background

$$
\begin{aligned}
d s^{2}= & \left(\frac{u}{R}\right)^{3 / 2}\left(\hat{f}(u) d t^{2}+\delta_{i j} d x^{i} d x^{j}+d \tau^{2}\right) \\
& +\left(\frac{R}{u}\right)^{3 / 2}\left(\frac{d u^{2}}{\hat{f}(u)}+u^{2} d \Omega_{4}^{2}\right), \\
\hat{f}(u)= & 1-\frac{u_{T}^{3}}{u^{3}},
\end{aligned}
$$

has a cigar-shaped $(u, t)$ subspace and a cylinder-shaped $(u, \tau)$ subspace, now with the periodicity of the $\tau$ circle arbitrary, but the periodicity of the $t$ circle fixed to $\delta t=$ $\beta=T^{-1}=\frac{4 \pi}{3} R^{3 / 2} u_{T}^{-1 / 2}$. These two backgrounds are identical, modulo a redefinition of coordinates $t$ and $\tau$, when $\delta \tau$ equals $\beta$, which happens at the deconfinement transition temperature $T_{c}=\frac{3}{4 \pi} R^{-3 / 2} u_{K}^{1 / 2}$. In the deconfining phase ${ }^{2}$ the embedding of the flavor branes is no longer forced to be $\cup$ shaped, as the $(u, \tau)$ space is no longer cigar shaped. At a certain value of the temperature, $T_{\chi} \geq T_{c}$, it will become energetically favorable for the flavor branes to fall straight down instead of merging in a $\cup$ shape, indicating chiral symmetry restoration (see Fig. 2). The essential features of QCD, chiral symmetry breaking and confinement, plus the ensuing chiral restoration and deconfinement at sufficiently large temperatures are thus nicely resembled by the SSM, among other QCD phenomenology [18]. To make further explicit contact with QCD, we determine the string related parameters of the SSM in physical $(\mathrm{GeV})$ units, so that an explicit comparison with other approaches comes within range.

\section{B. Fixing holographic parameters at $\boldsymbol{e B}=\mathbf{0}$}

In [18] the independent parameters $M$ and $\kappa$ of the antipodal $\left(u_{0}=u_{K}\right)$ SSM were fixed to $M \approx 0.949 \mathrm{GeV}$

\footnotetext{
${ }^{2}$ Let us remark here that in [24] some problems concerning the identification of the deconfined phase with the black D4-brane background were discussed, and instead a different background was proposed, namely a localized D3-soliton geometry. To make calculations of the critical temperatures feasible, it is, however, necessary to consider a high-temperature approximation of that background. There are moreover some subtleties concerning the inclusion of flavor branes, but the end result for the $(T, L)$ phase diagram (see Fig. 10 of [24]) gives a qualitatively similar result as the "old" SSM. Based on this observation, we can expect that qualitative features of the $e B$ dependence of the chiral transition temperatures are not unlikely to be similar in both backgrounds. It would be interesting to check this explicitly, but for the reasons mentioned above we will consider the simpler black D4-brane background in this paper. We thank Takeshi Morita for discussion on this point.
}

and $\kappa=\lambda /\left(72 \pi^{3}\right) \approx 0.00745$, with $\lambda=g_{Y M}^{2} N_{c}$ the 't Hooft coupling, by matching to the QCD input values

$$
f_{\pi}=0.093 \mathrm{GeV} \text { and } m_{\rho}=0.776 \mathrm{GeV}
$$

for the pion decay constant $f_{\pi}$ and the $\rho$ meson mass $m_{\rho}$. Because of the relations $u_{K}=M^{-1}, R^{3}=\frac{9}{4} M^{-3}$, and $g_{s}^{-1} \ell_{s}^{-3}=\frac{4 \pi}{3} M^{3}$ [18] all other parameters of the model are then fixed as well. Note that throughout this paper we set $N_{c}=3$.

In the non-antipodal case $\left(u_{0}>u_{K}\right)$ the matching conditions (3) do not fix all freedom, since there is one extra parameter present, $u_{0}$. From the eigenvalue equation that determines $m_{\rho}$ holographically,

$$
\begin{aligned}
\partial_{z}\left(\frac{3}{u_{0}} u_{z}^{1 / 2} \gamma^{\prime-1} \partial_{z} \psi_{\rho}\right) & =-\frac{4}{3} u_{0} u_{z}^{3 / 2} \gamma^{\prime} R^{3} m_{\rho}^{2} \psi_{\rho}, \\
\psi_{\rho}^{\prime}(0) & =0, \quad \psi_{\rho}( \pm \infty)=0,
\end{aligned}
$$

with

$$
\begin{aligned}
\gamma^{\prime}(z) & =\sqrt{\frac{z^{2}}{u_{z}^{5}\left(u_{z}^{3}-u_{K}^{3}\right)-\left(u_{0}^{8}-u_{0}^{5} u_{K}^{3}\right)^{\prime}}} \\
u_{z}^{3} & =u_{0}^{3}+u_{0} z^{2}
\end{aligned}
$$

we extract the values of $u_{0}$ that, for a given $M$, lead to $m_{\rho}=0.776 \mathrm{GeV}$. The resulting function $u_{0}(M)$ is plotted in Fig. 3 for a range of $M$-the maximum value of $M$ corresponding to the limiting case $u_{0} \rightarrow u_{K}=1 / M-$ alongside the function $L(M)$ for the corresponding asymptotic separation between branes and antibranes, determined from

$$
L=\int_{u_{0}}^{\infty} d u\left(\frac{R}{u}\right)^{3 / 2} f^{-1} \sqrt{\frac{u_{0}^{8} f_{0}}{u^{8} f-u_{0}^{8} f_{0}}},
$$

with $f\left(u_{0}\right)$ denoted as $f_{0}$. Next, demanding that the SSM prediction for the pion decay constant [25],

$f_{\pi}\left(M, u_{0}, \kappa\right)=\sqrt{\frac{4}{3} \kappa M^{7 / 2} \frac{3}{u_{0}}\left(2 \int_{0}^{\infty} d z \frac{\gamma^{\prime}}{\left(u_{0}^{3}+u_{0} z^{2}\right)^{1 / 6}}\right)^{-1}}$,

equals $0.093 \mathrm{GeV}$ leads to the function $\kappa(M)$ of allowed values for $\kappa$, as plotted in Fig. 3(c). The string tension $\left(2 \pi \alpha^{\prime}\right)^{-1}=8 \pi^{2} M^{2} \kappa(M)$ is then also known as a function of $M$.

The remaining freedom of choosing the mass scale $M$ can be fixed, for example, by matching the SSM prediction for the constituent quark mass,

$$
m_{q}\left(M, u_{0}, \kappa\right)=8 \pi^{2} M^{2} \kappa \int_{1 / M}^{u_{0}} d u \frac{1}{\sqrt{1-\frac{1}{(M u)^{3}}}},
$$

to a phenomenologically reasonable value, as can be read off from Fig. 4. 

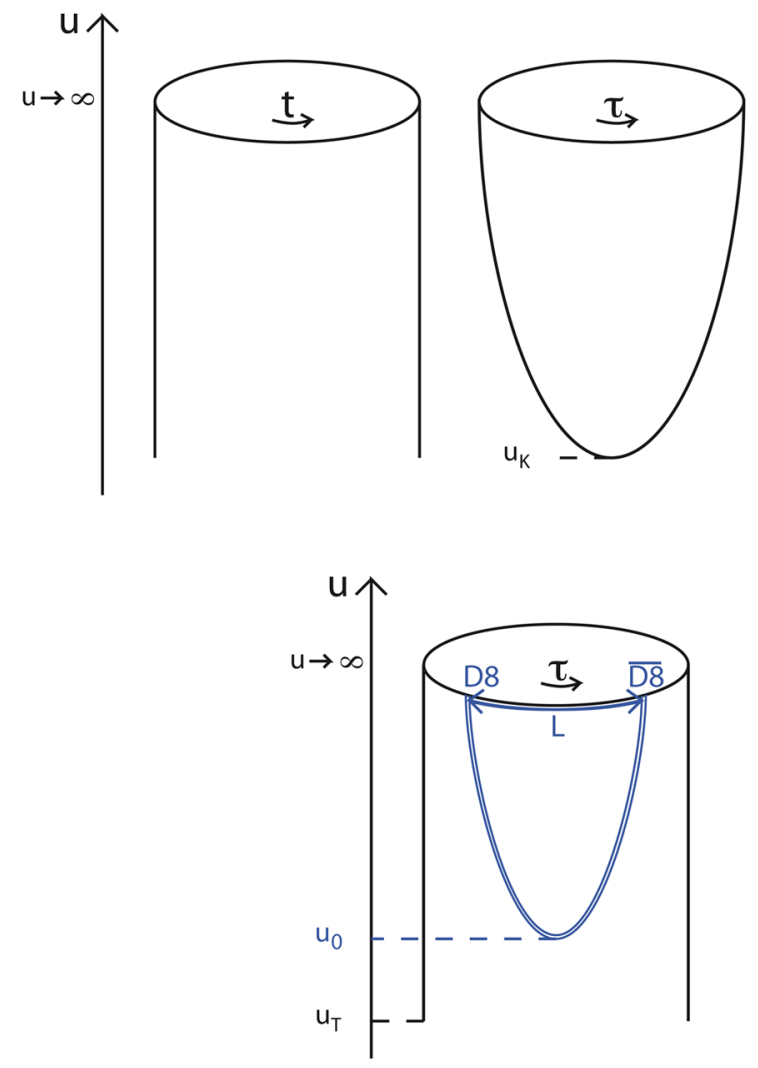
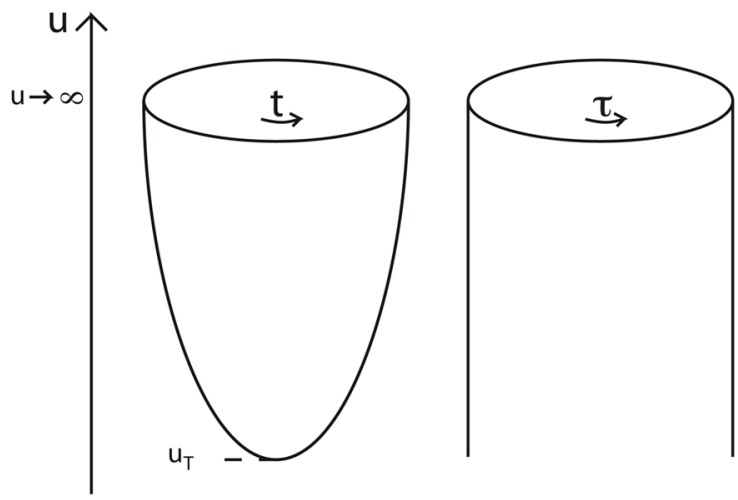

(a)

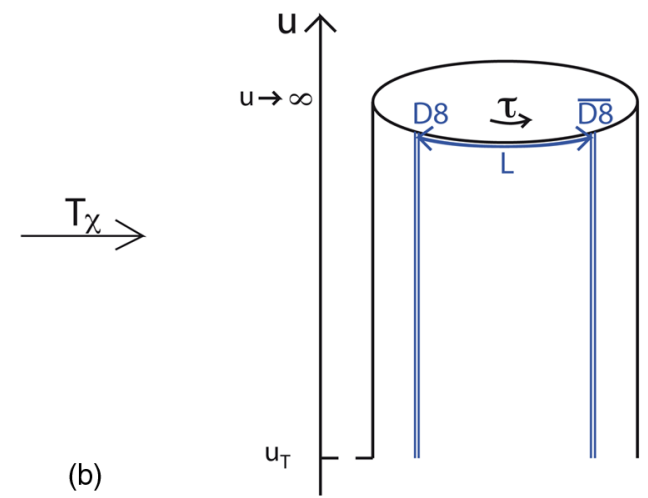

FIG. 2 (color online). (a) Deconfinement transition at $T_{c}$ and (b) chiral symmetry restoration at $T_{\chi}\left(\geq T_{c}\right)$.

In [25] we opted to reproduce $m_{q}=0.310 \mathrm{GeV}$, leading to the following set of fixed holographic parameters:

$$
\begin{aligned}
M & \approx 0.7209 \mathrm{GeV}, \\
L & \approx 1.574 \mathrm{GeV}^{-1}, \quad \text { and } \\
\kappa & =\frac{\lambda N_{c}}{216 \pi^{3}} \approx 0.006778 .
\end{aligned}
$$

With these values, the effective QCD string tension is computed to be $\sigma \approx 0.19 \mathrm{GeV}^{2}$ [25], in excellent agreement with the standard lattice value, $\sigma \approx 0.18-0.19 \mathrm{GeV}^{2}$, extracted from [26]. Said otherwise, we could equally well have selected the string tension as our third QCD input value instead of the constituent quark mass.

Here, we will, however, opt to leave $M$ variable, or equivalently $L$ via Fig. 3(b), with the eye on drawing the $(T, L, e B)$ phase diagram later, and, more importantly, with the idea that the choice of $M$ or $L$ should be left free, as it determines the choice of holographic theory: $L$ very small ( $\sim \delta \tau=\frac{2 \pi}{M}$ large $\sim M$ small) corresponding to an NJLtype boundary field theory $[23,27,28]$ versus $L=\delta \tau / 2$ maximal ( $\sim M$ maximal) corresponding to a maximal probing of the gluon background, i.e. the original antipodal SSM.

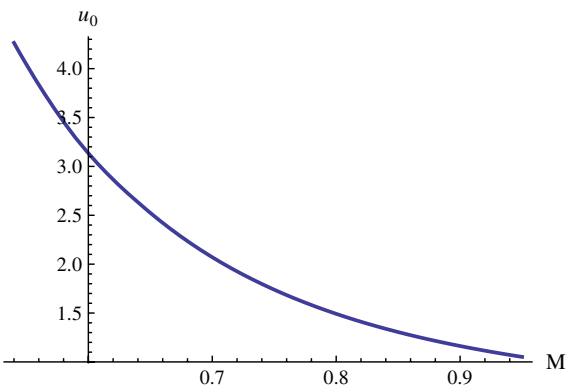

(a)

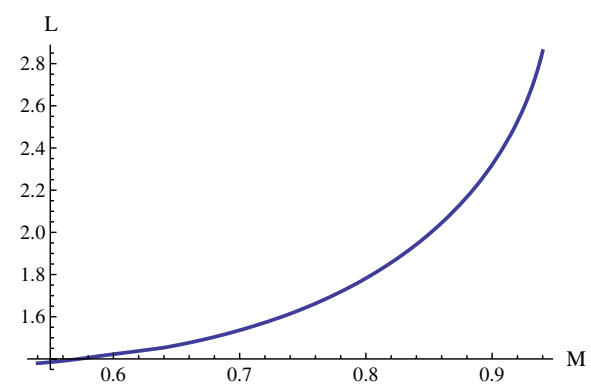

(b)

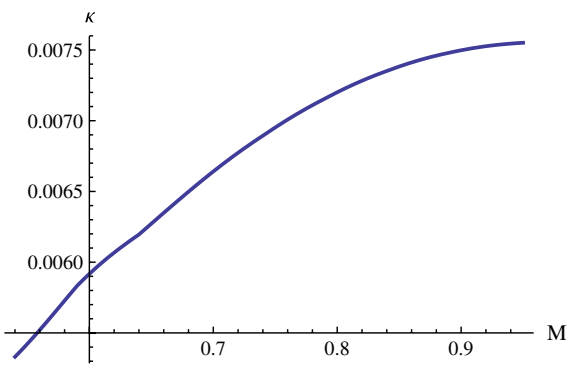

(c)

FIG. 3 (color online). (a) Values of $u_{0}\left(\mathrm{GeV}^{-1}\right)$ and (b) corresponding values of $L\left(\mathrm{GeV}^{-1}\right)$ compatible with $m_{\rho}=0.776 \mathrm{GeV}$. (c) Values of $\kappa$ compatible with $m_{\rho}=0.776 \mathrm{GeV}$ and $f_{\pi}=0.093 \mathrm{GeV}$. 


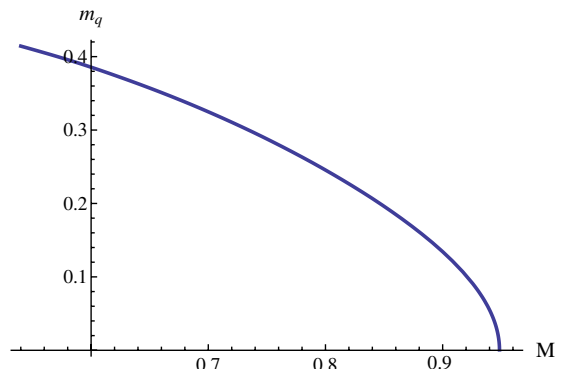

FIG. 4 (color online). SSM prediction for $m_{q}(\mathrm{GeV})$ as a function of the confinement scale $M(\mathrm{GeV})$, compatible with $m_{\rho}=0.776 \mathrm{GeV}$ and $f_{\pi}=0.093 \mathrm{GeV}$.

\section{Applying a magnetic field}

The next step is to introduce a magnetic background. To this purpose, we take a closer look at the $U\left(N_{f}\right)$ gauge field $A_{m}\left(x^{\mu}, u\right)(m=0,1,2,3, u)$ living on the D8-brane, with the non-Abelian Dirac-Born-Infeld (DBI) action ${ }^{3}$ [29]

$$
\begin{aligned}
S= & -2 T_{8} \int d^{4} x d u \\
& \times \int \epsilon_{4} e^{-\phi} \mathrm{S} \operatorname{Tr} \sqrt{-\operatorname{det}\left[g_{m n}^{D 8}+\left(2 \pi \alpha^{\prime}\right) i F_{m n}\right]},
\end{aligned}
$$

where the factor 2 in front makes sure that we integrate (in $u$ ) over both halves of the $U$-shaped D8-branes, $e^{\phi}=$ $g_{s}(u / R)^{3 / 4}, \epsilon_{4}$ is the volume form of the unit 4-sphere in the background, $T_{8}=1 /\left((2 \pi)^{8} \ell_{s}^{9}\right)$ is the D8-brane tension, $\mathrm{S} \operatorname{Tr}$ is the symmetrized trace defined as $\mathrm{S} \operatorname{Tr}\left(F_{1} \ldots F_{n}\right)=$ $\frac{1}{n !} \operatorname{Tr}$ (permutations of $\left.F_{1} \ldots F_{n}\right), g_{m n}^{D 8}$ is the induced metric on the D8-branes, $\alpha^{\prime}=\ell_{s}^{2}$ is the string tension, and $F_{m n}$ is the usual field strength; we use anti-Hermitian generators. As explained in $[18,25]$, one can slightly gauge the global $U\left(N_{f}\right)_{V}$ symmetry in the boundary field theory, i.e. make $g(u \rightarrow \infty) \in U\left(N_{f}\right)_{V} x$ dependent, so that $g \partial_{\mu} g^{-1} \equiv$ $\bar{A}_{\mu}(u \rightarrow \infty)$ corresponds to adding a background $U\left(N_{f}\right)_{V}$ field. Working in the $A_{u}=0$ gauge, the flavor gauge field can then be expanded as [18]

$$
A_{\mu}\left(x^{\mu}, u\right)=\bar{A}_{\mu}\left(x^{\mu}\right)+\text { rest, }
$$

where "rest" refers to pions and vector mesons in the boundary QCD theory, which are irrelevant for the current purposes.

An electromagnetic background field $A_{\mu}^{\mathrm{em}}$ can be switched on through

$$
\begin{aligned}
\bar{A}_{\mu} & =-i e \mathbf{Q}_{\mathrm{em}} A_{\mu}^{\mathrm{em}}=-i e\left(\begin{array}{cc}
2 / 3 & 0 \\
0 & -1 / 3
\end{array}\right) A_{\mu}^{\mathrm{em}} \\
& =-i e\left(\frac{\mathbf{1}_{2}}{6}+\frac{\sigma_{3}}{2}\right) A_{\mu}^{\mathrm{em}},
\end{aligned}
$$

\footnotetext{
${ }^{3}$ As the 't Hooft coupling $\lambda$ is large, we ignore the ChernSimons part of the action in the analysis, being a factor $1 / \lambda$ smaller than the DBI action.
}

with $\mathbf{Q}_{\mathrm{em}}$ the charge matrix for the up and down quarks. The choice $A_{\mu}^{\mathrm{em}}=x_{1} B \delta_{\mu 2}$ or $\bar{A}_{2}^{3}=x_{1} e B, \bar{A}_{2}^{0}=\bar{A}_{2}^{3} / 3$, ensures the desired constant magnetic background $\mathbf{B}=B \mathbf{e}_{3}$. The corresponding field strength tensor reads

$$
\bar{F}_{12}=\partial_{1} \bar{A}_{2}=-i\left(\begin{array}{cc}
\frac{2}{3} e B & 0 \\
0 & -\frac{1}{3} e B
\end{array}\right) \equiv-i\left(\begin{array}{cc}
\bar{F}_{u} & 0 \\
0 & \bar{F}_{d}
\end{array}\right) \text {. }
$$

\section{RESULTS}

\section{A. $e B$-dependent embedding in confinement phase}

For completeness, we briefly summarize here the discussion of the $e B$-dependent embedding of the $N_{f}=2$ flavor branes in the confinement phase, i.e. the determination of $u^{\prime}=d u / d \tau$ for each flavor, presented in more detail in [25].

The induced metric on the flavor branes is given by

$$
\begin{aligned}
d s_{D 8}^{2}= & g_{m n}^{D 8} d x^{m} d x^{n} \quad(m, n=0 \ldots 8) \\
= & \left(\frac{u}{R}\right)^{3 / 2} \eta_{\mu \nu} d x^{\mu} d x^{\nu}+\left(\left(\frac{R}{u}\right)^{3 / 2} \frac{1}{f(u)}+\left(\frac{u}{R}\right)^{3 / 2} \frac{f(u)}{u^{\prime 2}}\right) d u^{2} \\
& +\left(\frac{R}{u}\right)^{3 / 2} u^{2} d \Omega_{4}^{2},
\end{aligned}
$$

where the metric components in flavor space are assumed to be different, to allow for a different response of both flavor branes to the magnetic field:

$$
g^{D 8}=\left(\begin{array}{cc}
g_{u}^{D 8} & 0 \\
0 & g_{d}^{D 8}
\end{array}\right)
$$

with the $u$-coordinate appearing in $g_{u}^{D 8}\left(g_{d}^{D 8}\right)$ following the up (down) brane, thus $u \in\left[u_{0, u}, \infty\right]$ or $u \in\left[u_{0, d}, \infty\right]$. Inserting this metric ansatz into the DBI action (10), along with the $\bar{A}_{\mu}$ background, gives $S^{\text {conf }}=S_{u}+S_{d}$ with

$$
\begin{aligned}
& S_{\ell}=-T_{8} V_{4} V_{4} g_{s}^{-1} 2 \int_{u_{0, \ell}}^{\infty} d u u^{4} \sqrt{\frac{1}{f}\left(\frac{u}{R}\right)^{-3}+\frac{f}{u^{\prime 2}}} \sqrt{A_{\ell}}, \\
& A_{\ell}=1+\left(2 \pi \alpha^{\prime}\right)^{2} \bar{F}_{\ell}^{2}\left(\frac{R}{u}\right)^{3},
\end{aligned}
$$

where the index $\ell$ refers to the up or down flavor, $u^{\prime}=$ $d u / d \tau, V_{4}=\int d^{4} x$, and $V_{4}$ is the volume of the unit 4-sphere. The DBI action for the two D8-branes thus reduces to the sum of two Abelian actions, which is the geometric translation of the explicit breaking of chiral symmetry $U(2)_{A} \stackrel{e B}{\rightarrow} U(1)_{A}^{u} \times U(1)_{A}^{d}$.

Suppressing the $\ell$ index for the moment, $u^{\prime}$ can be determined from the conserved "Hamiltonians" 


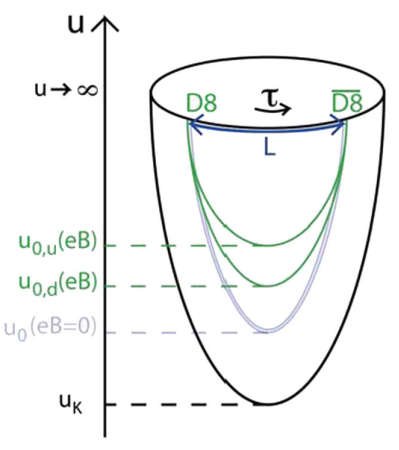

(a)

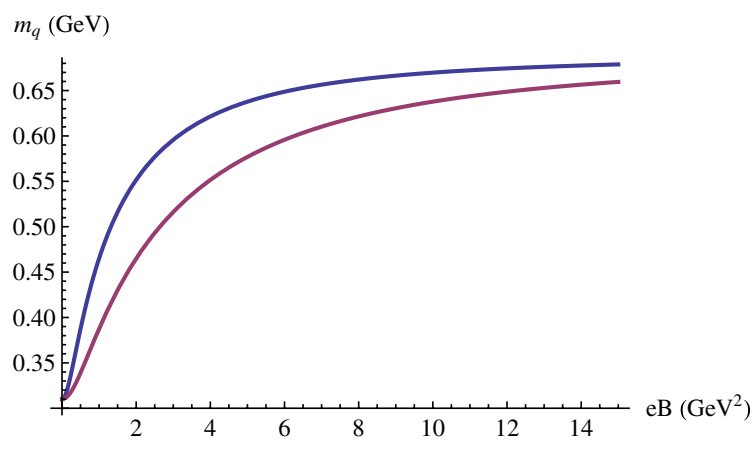

(b)

FIG. 5 (color online). (a) Up and down flavor brane embedding in the presence of a magnetic field, (b) corresponding constituent quark masses $m_{q, u}$ (blue curve) and $m_{q, d}$ (red curve) for parameter choice (9).

$$
\begin{aligned}
H & =u^{\prime} \frac{\delta \mathcal{L}^{\tau}}{\delta u^{\prime}}-\mathcal{L}^{\tau}=\frac{-u^{4} f \sqrt{A}}{\sqrt{\frac{u^{\prime 2}}{f}\left(\frac{u}{R}\right)^{-3}+f}}, \quad \partial_{\tau} H=0, \text { with } \\
\mathcal{L}^{\tau} & =u^{4} \sqrt{\frac{u^{\prime 2}}{f}\left(\frac{u}{R}\right)^{-3}+f \sqrt{A},}
\end{aligned}
$$

by assuming $\cup$-shaped embeddings, $u^{\prime}=0$ at $u=u_{0}$ [with $A\left(u_{0}\right)$ and $f\left(u_{0}\right)$ denoted as $A_{0}$ and $f_{0}$, for each flavor]. In the confinement phase, the $e B$-dependent action on each flavor brane is then given by

$$
\begin{aligned}
S_{\ell} & =-T_{8} V_{4} V_{4} g_{s}^{-1} 2 \int_{u_{0, \ell}}^{\infty} d u R^{3 / 2} u^{5 / 2} \sqrt{A_{\ell}} \sqrt{\gamma_{B, \ell}}, \\
\gamma_{B, \ell}(u) & =\frac{u^{8} A_{\ell}}{u^{8} f A_{\ell}-u_{0, \ell}^{8} f_{0} A_{0, \ell}},
\end{aligned}
$$

and the asymptotic separations $L=2 \int d u / u^{\prime}$ become (suppressing $\ell$ for notational convenience)

$$
\begin{aligned}
& L^{\operatorname{conf}}\left(u_{0}, e B\right)=2 \int_{u_{0}}^{\infty} d u\left(\frac{R}{u}\right)^{3 / 2} f^{-1} \sqrt{\frac{u_{0}^{8} f_{0} A_{0}}{u^{8} f A-u_{0}^{8} f_{0} A_{0}}} \\
& \zeta=\left(u / u_{0}\right)^{-3} \\
& \stackrel{2}{\frac{2}{3}} \frac{R^{3 / 2}}{\sqrt{u_{0}}} \sqrt{f_{0} A_{0}} \int_{0}^{1} d \zeta \frac{f^{-1} \zeta^{1 / 2}}{\sqrt{f A-f_{0} A_{0} \zeta^{8 / 3}}} .
\end{aligned}
$$

To model magnetic catalysis, we keep $L^{\operatorname{conf}}\left(u_{0}, e B\right)$ fixed, for each flavor, at its flavor-independent value $L$ for $e B=0$. From the viewpoint of the boundary field theory, the flavor branes are extensive objects that stretch out infinitely far, viz. from $u=\infty$ to $u=u_{0}$ into the higher-dimensional bulk space. As such, from this asymptotic perspective, it would appear that it would cost an infinite amount of energy to move the branes at $u=\infty$. Keeping $L$ fixed thus seems to be a sensible boundary condition, one also used in e.g. [27,30], to probe the effects of the bulk dynamics in the presence of the external field. The value of $L$, ranging from $\frac{\delta \tau}{2}$ to negligible with respect to $\frac{\delta \tau}{2}$, determines how much of the bulk dynamics is probed, ranging, respectively, from all to none.

So, as we keep $L^{\text {conf }}$ fixed to its value $L$ at zero magnetic field, from here on also explicitly writing the $M$ dependence of $L^{\text {conf }}$ through $R$ and $2 \pi \alpha^{\prime}$, we can extract the $e B$ dependence of $u_{0}$ and of the corresponding constituent quark masses $m_{q, u}$ and $m_{q, d}$, which correspond to the mass (8) of a string stretched between one of the flavor branes and the cutoff at $u_{K}=M^{-1}$ [23]:

$L_{\ell}^{\operatorname{conf}}\left(u_{0}, e B, M\right)=L \Rightarrow u_{0, \ell}(e B, L, M) \Rightarrow m_{q, \ell}(e B, L, M)$.

Using the correspondence between $L$ and $M$ as plotted in Fig. 3(b) such that $m_{\rho}=0.776 \mathrm{GeV}$, we obtain $m_{q}(e B, M)$ or $m_{q}(e B, L)$. This turns out to be a rising function of $e B$ for all choices of $M$ or $L$, modeling chiral magnetic catalysis, as already noted in [22] for the single-flavor case. The magnetic field thus indeed boosts the chiral symmetry breaking, reflected in a stronger bending of the flavor branes, and the breaking is stronger for the up than for the down flavor; see Fig. 5(a). This was expected, since the up quark couples twice as strong to the magnetic field. For the particular choice of parameters (9), $m_{q}$ is plotted in Fig. 5(b). Both constituent masses show a quadratic dependence on $e B$ for small magnetic fields, which then becomes nearly linear and eventually thrives to saturation at very large $e B$. This linear behavior is in relative agreement with recent lattice estimates of the chiral condensate ${ }^{4}$ in $[10,14,21]$. A linear behavior, inspired by chiral perturbation theory, was fitted to the SU(2) lattice data for the chiral condensate in [31], while SU(3) data was fitted with a $(e B)^{3 / 2}$ behavior in [32]; both these studies used one quenched flavor. Let us, however, point out that the plots of the lattice chiral condensates in all cited works do seem to display a nontrivial curvature for small values of $e B$.

\footnotetext{
${ }^{4}$ The concept of the chiral condensate is not well defined in the SSM; we therefore use the constituent quark mass as a measure for the chiral symmetry breaking.
} 
The stability of the flavor branes' embedding as shown in Fig. 5(a) is discussed in [25]. At sufficiently low values of $e B$, it is stable, but at higher values of $e B$, we should not necessarily trust our findings, as an instability could occur in the $\rho$ meson sector at $e B \sim m_{\rho}^{2} \sim 30 m_{\pi}^{2}\left(\approx 0.6 \mathrm{GeV}^{2}\right)$, driving a condensation [33]. Evidently, this would lead to a different underlying action, describing the theory in the condensed phase. If this happens, also other models should take it into account.

\section{B. $e B$-dependent embedding in deconfinement phase}

Next, we turn to the finite temperature case. The background at $T<T_{c}$ is identical to the zero temperature background up to the period $\beta=T^{-1}$ of Euclidean time, so nothing changes as compared to the $T=0$ case. Things get more interesting once we enter the deconfinement region. We again have an induced metric on each flavor brane,

$$
\begin{aligned}
d s_{\mathrm{D} 8}^{2}= & \left(\frac{u}{R}\right)^{3 / 2}\left(\hat{f} d t^{2}+\delta_{i j} d x^{i} d x^{j}\right)+\left(\frac{R}{u}\right)^{3 / 2} u^{2} d \Omega_{4}^{2} \\
& +\left(\frac{u}{R}\right)^{3 / 2}\left[\frac{1}{\hat{f}}\left(\frac{u}{R}\right)^{-3}+\frac{1}{u^{\prime 2}}\right] d u^{2},
\end{aligned}
$$

with periodicity of the $t$ circle given by

$$
\delta t=T^{-1}=\frac{4 \pi}{3} R^{3 / 2} u_{T}^{-1 / 2},
$$

from which one can determine the action in the deconfined phase, completely analogous to the derivation of the action in the confined phase. For temperatures $T<T_{\chi, \ell}$, the $\ell$-brane's embedding remains $\cup$ shaped, with action

$$
S_{\ell}^{T<T_{\chi \ell \ell}}=c_{0} u_{0, \ell}^{7 / 2} \int_{1}^{\infty} d y y \sqrt{y^{3} A_{\ell}} \sqrt{\frac{1}{1-\frac{\hat{f}_{0, \ell} A_{0 \ell}}{\hat{f}_{\ell}(y) y^{3} A_{\ell}} y^{-5}}},
$$

where $\quad c_{0}=-2 T_{8} V_{4} V_{4} g_{s}^{-1} R^{3 / 2}, \quad y=u / u_{0, \ell}, \quad y_{T, \ell}=$ $u_{T} / u_{0, \ell}, \quad \hat{f}_{\ell}(y)=1-\left(y_{T, \ell} / y\right)^{3}, \quad$ and $\quad \hat{f}_{0, \ell}=1-y_{T, \ell}^{3}$.

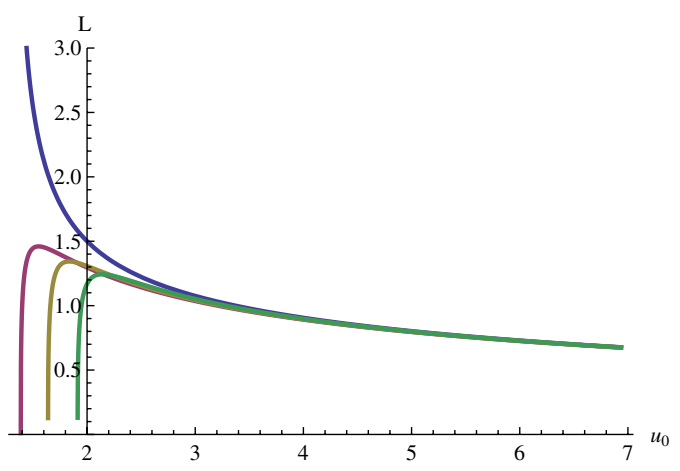

(a)
If $T>T_{\chi, \ell}$, the $\ell$-branes are falling straight down, $u^{\prime}=$ $\infty$, with action

$$
S_{\ell}^{T>T_{\chi, \ell}}=c_{0} u_{0, \ell}^{7 / 2} \int_{y_{T, \ell}}^{\infty} d y y \sqrt{y^{3} A_{\ell}} .
$$

The chiral transition temperature $T_{\chi, \ell}$ is the temperature for which $\Delta S_{\ell}$ becomes zero [23], with

$$
\Delta S\left(u_{0}, e B, y_{T}\right)=\text { action }_{\cup \text {-shape }}-\text { action }_{\text {straight }} .
$$

The correspondence between $u_{0}$ and $L$ in the deconfined phase is modified into (again suppressing the flavor index here)

$L^{\mathrm{dec}}\left(u_{0}, e B, y_{T}\right)=\frac{2}{3} \frac{R^{3 / 2}}{\sqrt{u_{0}}} \sqrt{\hat{f}_{0} A_{0}} \int_{0}^{1} d \zeta \frac{\hat{f}^{-1 / 2} \zeta^{1 / 2}}{\sqrt{\hat{f} A-\hat{f}_{0} A_{0} \zeta^{8 / 3}}}$.

As before, we will hold the asymptotic separation fixed at its starting value $L$ at $e B=0$ and $T=0$. This allows us to determine the $e B$ and $T$ dependence of $u_{0}$ (from here on also explicitly writing the dependence on $M$, through $R$ and $\left.2 \pi \alpha^{\prime}\right)$ :

$$
L^{\mathrm{dec}}\left(u_{0}, e B, y_{T}, M\right)=L \Rightarrow u_{0}\left(e B, y_{T}, L, M\right) .
$$

From Fig. 6(a) it can be seen that the one-to-one correspondence between $u_{0}$ and $L^{\text {conf }}$ is not preserved in the deconfinement phase, where each value of $L^{\text {dec }}$ corresponds to two possible values of $u_{0}$, as long as it does not exceed its maximum possible value (i.e. as long as $T<T_{\chi}$ ). We numerically verified that the energetically favored solution for $u_{0}$ is the largest one, consistent with the intuition that the lower- $u_{0}$ solution contains more energy as it probes a larger portion of the background. Keeping $L$ fixed during the deconfinement transition causes a jump in $u_{0}$, as well as in the constituent quark and meson masses [30].

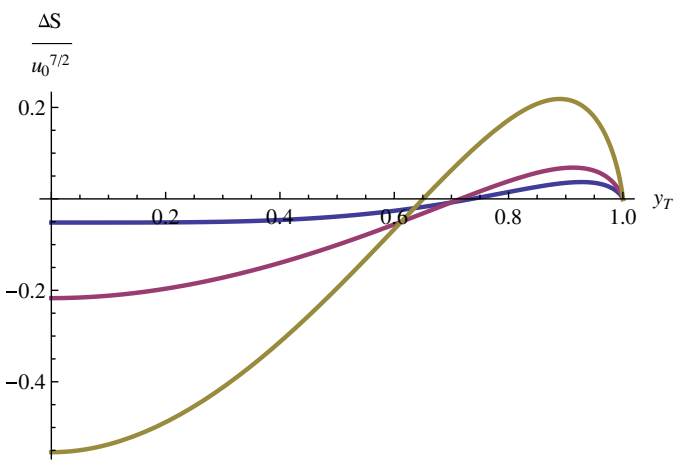

(b)

FIG. 6 (color online). (a) $L^{\text {conf }}$ (blue curve) and $L^{\mathrm{dec}}\left(\mathrm{GeV}^{-1}\right)$ for $T=T_{c}$ (red curve) and increasing values of $T\left(>T_{c}\right.$ ) at $e B=0$, (b) $\Delta S / u_{0}^{7 / 2}$ as a function of $y_{T}$ for $e B=0$ (blue curve), 0.5 (purple curve), and $1.6 \mathrm{GeV}^{2}$ (yellow curve). Both figures for $M=0.7209 \mathrm{GeV}$. 

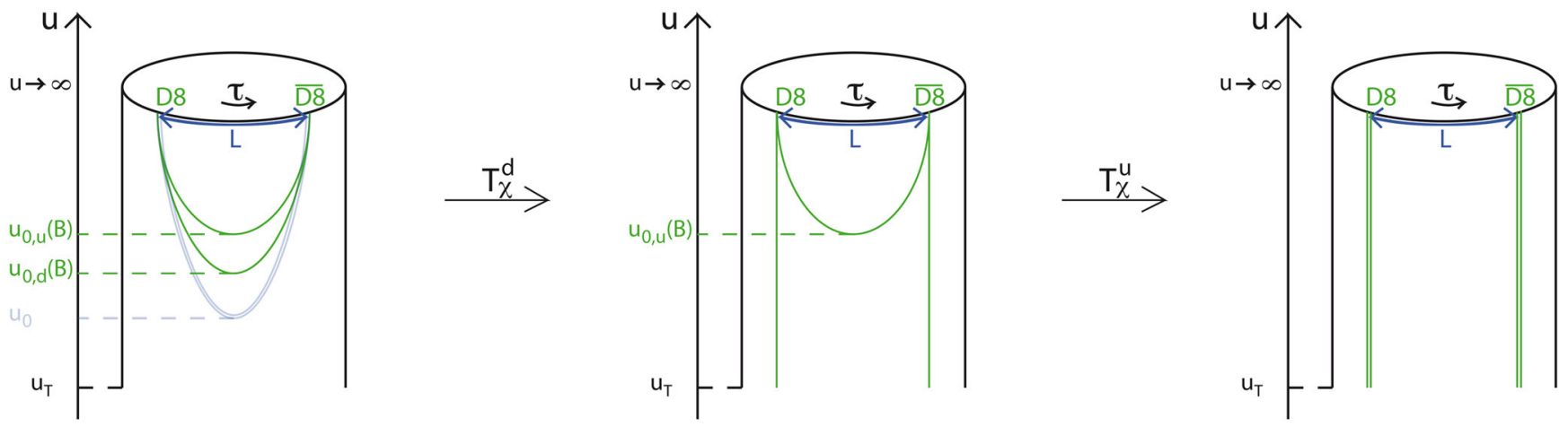

FIG. 7 (color online). Embeddings in the deconfined phase with magnetic field.

With the expression found for $u_{0}$, the expression for $\Delta S$ at fixed $L$ is also known,

$\Delta S\left(u_{0}\left(e B, y_{T}, L, M\right), e B, y_{T}, M\right) \equiv \Delta S\left(e B, y_{T}, L, M\right)$

so the chiral temperature can be determined from the point where the U-shaped embedding breaks into separated branes, i.e. when $\Delta S=0$ [see Fig. 6(b)],

$$
\Delta S\left(e B, y_{T}, L, M\right)=0 \Rightarrow y_{T}^{\chi}(e B, L, M) .
$$

The corresponding value of $u_{0}$ at the chiral transition is then given by

$$
u_{0}^{\chi}=u_{0}\left(e B, y_{T}^{\chi}(e B, L, M), L, M\right) \equiv u_{0}^{\chi}(e B, L, M) .
$$

Plugging the obtained $y_{T}^{\chi}(e B, L, M)$ and $u_{0}^{\chi}(e B, L, M)$ into the definition for the chiral temperature

$$
\begin{aligned}
T_{\chi} & =\frac{3}{4 \pi} \sqrt{\frac{u_{T}^{\chi}}{R^{3}}}=\frac{3}{4 \pi} \sqrt{y_{T}^{\chi}} \sqrt{\frac{u_{0}^{\chi}}{R^{3}}} \\
& =\frac{3}{4 \pi} \sqrt{y_{T}^{\chi}(e B, L, M)} \sqrt{\frac{u_{0}^{\chi}(e B, L, M)}{R^{3}}} \equiv T_{\chi}(e B, L, M),
\end{aligned}
$$

we obtain $T_{\chi}(e B, L, M)$.

From the parameter discussion at $e B=0$ we know the value of the fixed asymptotic separation given a value for $M$ such that $m_{\rho}=0.776 \mathrm{GeV}$ [Fig. 3(b)]; hence we obtain $T_{\chi}(e B, M)$ or $T_{\chi}(e B, L)$, to be compared with the deconfinement temperature $T_{c}=M /(2 \pi)$. The deconfinement temperature $T_{c}$ will not change as it is determined from the background D4-brane metric, which could only become $e B$ dependent when the backreaction of the D8-branes would be taken into account. Or, field theoretically, $T_{c}$ is $e B$ independent in a quenched setup, because the magnetic field can only couple to the neutral gluons indirectly via the quark interactions. For every choice of $M$ (or $L$ ), $T_{\chi}(e B)$ rises with $e B$ ("chiral magnetic catalysis"). But depending on the choice, there will or will not arise a split between $T_{\chi}$ and $T_{c}$. Doing this for each flavor, we find $T_{\chi}^{u}(e B, L)$ and $T_{\chi}^{d}(e B, L)$, with $T_{\chi}^{u}$ consistently higher than $T_{\chi}^{d}$ for a given value of $L$, as expected. This leads to an intermediate phase where the chiral symmetry for up quarks is still broken while the chiral symmetry for down quarks is already restored:

$$
\begin{aligned}
& U(1)_{V}^{u} \times U(1)_{V}^{d} \stackrel{T_{X}^{d}}{\rightarrow} U(1)_{V}^{u} \times\left(U(1)_{L} \times U(1)_{R}\right) \stackrel{T^{\prime}}{\rightarrow}\left(U(1)_{L}\right. \\
& \left.\quad \times U(1)_{R}\right)^{u} \times\left(U(1)_{L} \times U(1)_{R}\right)^{d},
\end{aligned}
$$

as sketched in Fig. 7.

In Fig. 8 the $(T, M, e B)$ and $(T, L, e B)$ phase diagrams of the two-flavor non-antipodal SSM are plotted. This generalizes the $N_{f}=1 \mathrm{SSM}$ phase diagram in Fig. 7 of [23] to the $N_{f}=2$ magnetic case. For setups with large values of $M$, namely $M>0.767 \mathrm{GeV}$ corresponding to $m_{q}(e B=$ $0)<0.274 \mathrm{GeV}$ or $L>1.681 \mathrm{GeV}^{-1}$, there is no split between $T_{\chi}(e B, M)$ and $T_{c}=M /(2 \pi)$, no matter how large the applied magnetic field is. This is a consequence of the saturation of the rising of $T_{\chi}$ with $e B$. In a SSM with $M<0.657 \mathrm{GeV}$, corresponding to $m_{q}(e B=0)>$ $0.353 \mathrm{GeV}$ or $L<1.473 \mathrm{GeV}^{-1}$, there is already a split between chiral and deconfinement transition before the magnetic field is turned on: $T_{\chi}(e B=0, M)>T_{c}$, which becomes larger as $e B$ increases. This regime is probably the least physically relevant, as the values for constituent quark masses are too large and the values for the deconfinement temperature smaller than $0.105 \mathrm{GeV}$, which is rather small compared to the chiral limit value we can extrapolate "by hand" from [34], giving $T_{c} \sim 0.150 \mathrm{GeV}$. The third possible case is that the value of $M$ is such, $0.657 \mathrm{GeV}<$ $M<0.767 \mathrm{GeV} \quad\left[\sim 0.274 \mathrm{GeV}<m_{q}(e B=0)<0.353 \mathrm{GeV}\right.$ or $\left.1.473 \mathrm{GeV}^{-1}<L<1.681 \mathrm{GeV}^{-1}\right]$, that $T_{\chi}(e B=$ $0, M)=T_{c}$ but a split between $T_{\chi}$ and $T_{c}$ arises at some value of $e B$, plotted in Fig. 10. For each of the above possible cases, an exemplary cross section of the $(T, M, e B)$ phase diagram is shown in Fig. 9, the middle one corresponding to the best matching parameters for reproducing a reasonable $m_{q}(e B=0) \approx 0.310 \mathrm{GeV}$, although the corresponding value for $T_{c} \approx 0.115 \mathrm{GeV}$ is still on the small side. A deconfinement temperature $T_{c} \approx$ $0.150 \mathrm{GeV}$ would correspond to $M=0.942 \mathrm{GeV}$, very close to the antipodal value in the regime where no split 


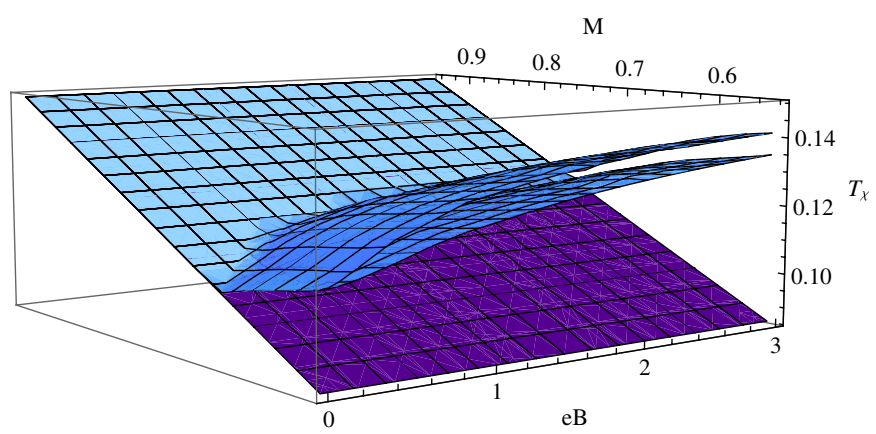

(a)

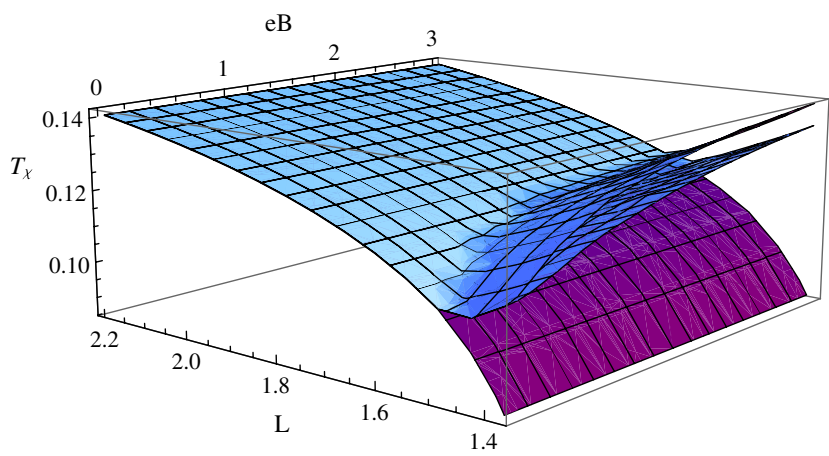

(b)

FIG. 8 (color online). (a) $T_{\chi}^{u}(\mathrm{GeV})$ (upper blue surface) and $T_{\chi}^{d}(\mathrm{GeV})$ (lower blue surface) as functions of $e B\left(\mathrm{GeV}{ }^{2}\right)$ and $M(\mathrm{GeV})$ compared to $T_{c}(M)(\mathrm{GeV})$ (purple surface), (b) same with $M$ dependence replaced by $L$ dependence compatible with $m_{\rho}=0.776 \mathrm{GeV}$.

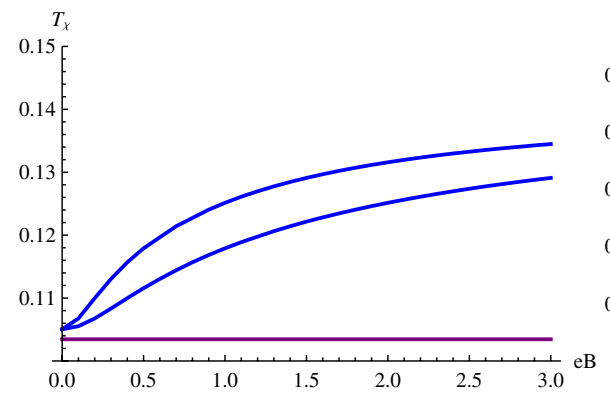

(a)

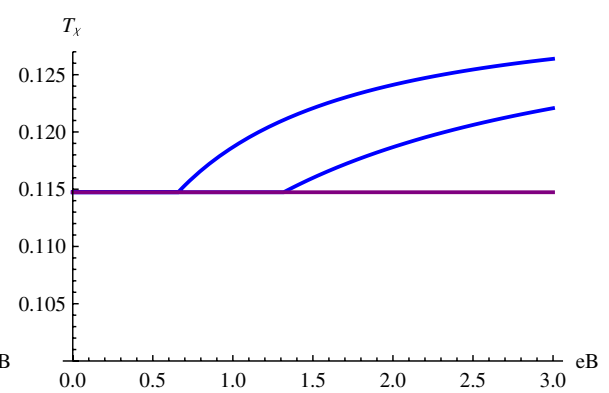

(b)

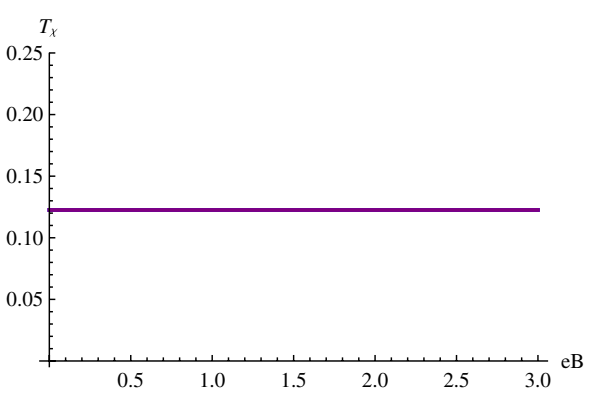

(c)

FIG. 9 (color online). Cross sections of Fig. 8 for (a) $M=0.65 \mathrm{GeV}$, (b) $M=0.7209 \mathrm{GeV}$, and (c) $M=0.77 \mathrm{GeV}$, respectively, corresponding to $m_{q}(e B=0)=0.357,0.310$, and $0.272 \mathrm{GeV}$ and $T_{c}=0.103,0.115$, and $0.123 \mathrm{GeV}$. The appearance of a split between $T_{\chi}(\mathrm{GeV})$ (blue curve) and $T_{c}(\mathrm{GeV})$ (purple curve) depends on the choice of $M$, or equivalently $L$.

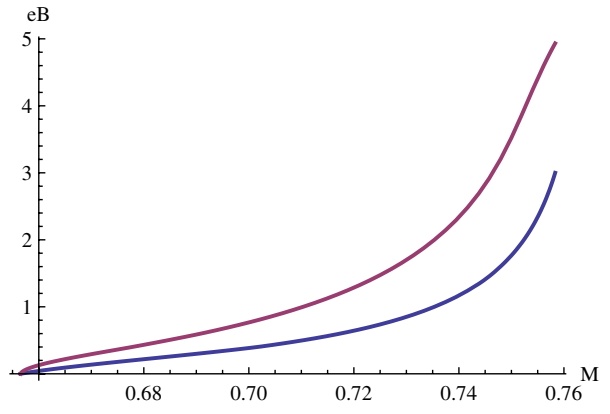

FIG. 10 (color online). Value of $e B$ where $T_{\chi}^{u}(\mathrm{GeV})$ (blue curve) $\left[T_{\chi}^{d}(\mathrm{GeV})\right.$ (purple curve) $]$ becomes larger than $T_{c}(\mathrm{GeV})$ for confinement scale values $0.657 \mathrm{GeV}<M<0.767 \mathrm{GeV}$.

arises between $T_{\chi}$ and $T_{c}$, leading, however, to an unphysically ${ }^{5}$ small $m_{q}(e B=0) \approx 0.046 \mathrm{GeV}$.

The papers [22,27] also pointed out the splitting of the critical temperatures for the one flavor version of the SSM,

\footnotetext{
${ }^{5}$ This might be related to the shortcoming of the SSM (in the used form, not considering possible modifications as in [20]) that the bare quark masses always remain zero.
}

but leaving the parameters of the SSM undetermined, making an explicit comparison with other approaches harsh. The explicit breaking of the global flavor symmetry by the different electromagnetic coupling of the up and down flavors is also now taken into account for the first time, leading to a split between the two chiral transitions themselves.

The fact that a split between $T_{\chi}$ and $T_{c}$ can emerge only for sufficiently small values of the asymptotic brane separation $L$, i.e. sufficiently close to an NJL effective description of QCD, seems to be supported by NJL model calculations [8] (see also the discussions in $[7,15,35]$ ) that seemingly contrast with lattice data. Selecting the holographic parameters in a way that brings the SSM as close as possible to (the chiral limit of) QCD, rather leads to a picture of the form of Figs. 9(b) or 9(c), namely no split at all or a small split that only emerges at rather large values of $e B$. Our findings are in this perspective consistent with lattice data of [21], where a split was not reported. However, we must also repeat here that our results are obtained in a quenched framework; hence important QCD effects at the level of transitions can be missing (e.g. pion loop effects). In particular, magnetic effects on 
the deconfinement temperature cannot be taken into account in the SSM without including backreaction of the probe branes on the background D4 metric.

\section{Remark on the antipodal SSM}

In the original antipodal Sakai-Sugimoto model, with $u_{0}=u_{K}$ and the asymptotic separation $L$ taking its maximum possible value, the embedding of the flavor branes is unaffected by the presence of the magnetic field. From this we can conclude that the antipodal Sakai-Sugimoto model is unable to capture the magnetically induced explicit breaking of chiral symmetry, as well as the chiral magnetic catalysis. Chiral symmetry restoration and deconfinement coincide for all values of the magnetic field.

\section{SUMMARY}

In conclusion, we have investigated the phase diagram of the two flavor version of the non-antipodal SakaiSugimoto model in the presence of a temperature $T$ and external magnetic field $e B$. In particular, we paid attention to fixing the holographic parameters, presenting a discussion of how they can be fixed by matching to carefully chosen QCD input parameters, in order to be able to present the phase diagram and related results in physical $\mathrm{GeV}$ units. This makes comparison to other approaches more direct. We indeed could compare our results with lattice and NJL results, the main conclusion being that the SSM results are consistent with other quenched settings that are able to model chiral magnetic catalysis.

The main results are presented in the $(T, L, e B)$ phase diagram in Fig. 8 and cross sections of that plot for different values of $L$ in Fig. 9. Here, $L$ is the asymptotic separation between the flavor probe branes. Keeping $L$ fixed serves as a boundary condition for the bulk dynamics, the effective boundary theory ranging from the NJL type for small $L$ to a chiral QCD-like theory where gluon dynamics are fully taken into account for maximal $L$. The value of $L$, i.e. the choice of the type of boundary model in a sense, determines if a split between the chiral and deconfinement temperature may or may not arise, as summarized in Fig. 9. Because of the different coupling to the magnetic background of the $u$ and $d$ flavor brane, we also find a split between the separate chiral transition temperatures.

It remains a challenge to construct a holographic dual of realistic QCD that could also describe the complicated finite temperature (above and below $T_{c}$ ) behavior of the chiral condensate as found in the latest lattice results [10]. This would require taking backreaction of the flavor branes on the background metric into account. A holographic model that could be interesting to look at from this point of view, is the Kuperstein-Sonnenschein model [36], in which chiral magnetic catalysis was observed [37] in the nonbackreacted case, and which was extended to include backreaction (be it perturbatively and for vanishing magnetic field) in [38], using a smearing technique. A downside of this model is, however, that it does not incorporate confinement due to the choice of the background in which the flavor branes are placed.

We end by noticing that the magnetic phase diagram could become even more intricate, as demonstrated in [33]: the QCD vacuum could become superconducting at sufficiently strong magnetic field. This matter is currently under investigation also in the holographic framework; see also $[25,39]$. We hope the presented results will stimulate further research in the area of QCD in a strong magnetic background, where many exciting new physics could be awaiting discovery. The gauge-gravity correspondence can offer viable input, next to QCD model and lattice based approaches.

\section{ACKNOWLEDGMENTS}

We thank the Research-Foundation Flanders (FWOVlaanderen) for financial support. We are grateful to D. Boer, E. Caceres, M. N. Chernodub, E. S. Fraga, A. J. Mizher, L. F. Palhares, A. Rebhan, A. Schmitt, and H. Verschelde for insightful discussions.
[1] T. Vachaspati, Phys. Lett. B 265, 258 (1991).

[2] D. E. Kharzeev, L. D. McLerran, and H. J. Warringa, Nucl. Phys. A803, 227 (2008).

[3] V. Skokov, A. Y. Illarionov, and V. Toneev, Int. J. Mod. Phys. A 24, 5925 (2009); A. Bzdak and V. Skokov, Phys. Lett. B 710, 171 (2012); W.-T. Deng and X.-G. Huang, Phys. Rev. C 85, 044907 (2012); K. Tuchin, arXiv:1301.0099.

[4] D. E. Kharzeev, K. Landsteiner, A. Schmitt, and H.-U. Yee, arXiv:1211.6245.

[5] Y. Aoki, G. Endrodi, Z. Fodor, S. D. Katz, and K. K. Szabo, Nature (London) 443, 675 (2006).

[6] A. Bazavov et al., Phys. Rev. D 80, 014504 (2009).
[7] A. J. Mizher, M. N. Chernodub, and E. S. Fraga, Phys. Rev. D 82, 105016 (2010); Proc. Sci., FacesQCD (2010) 020 [http:// pos.sissa.it/archive/conferences/117/020/FacesQCD_020.pdf].

[8] R. Gatto and M. Ruggieri, Phys. Rev. D 83, 034016 (2011); 82, 054027 (2010); K. Fukushima, M. Ruggieri, and R. Gatto, Phys. Rev. D 81, 114031 (2010); J. O. Andersen, J. High Energy Phys. 10 (2012) 005; N. O. Agasian and I. A. Shushpanov, Phys. Lett. B 472, 143 (2000).

[9] M. D'Elia, S. Mukherjee, and F. Sanfilippo, Phys. Rev. D 82, 051501 (2010).

[10] G. S. Bali, F. Bruckmann, G. Endrodi, Z. Fodor, S. D. Katz, S. Krieg, A. Schafer, and K. K. Szabo, J. High 
Energy Phys. 02 (2012) 044; G. S. Bali, F. Bruckmann, G. Endrodi, Z. Fodor, S. D. Katz, and A. Schafer, Phys. Rev. D 86, 071502 (2012).

[11] E. S. Fraga and L.F. Palhares, Phys. Rev. D 86, 016008 (2012); K. Fukushima and Y. Hidaka, Phys. Rev. Lett. 110, 031601 (2013).

[12] V. A. Miransky and I. A. Shovkovy, Phys. Rev. D 66, 045006 (2002).

[13] F. Bruckmann, G. Endrodi, and T. G. Kovacs, J. High Energy Phys. 04 (2013) 112.

[14] M. D'Elia and F. Negro, Phys. Rev. D 83, 114028 (2011).

[15] J. K. Boomsma and D. Boer, Phys. Rev. D 81, 074005 (2010).

[16] A. P. Mackenzie and Y. Maeno, Rev. Mod. Phys. 75, 657 (2003).

[17] J. M. Maldacena, Adv. Theor. Math. Phys. 2, 231 (1998).

[18] T. Sakai and S. Sugimoto, Prog. Theor. Phys. 113, 843 (2005); 114, 1083 (2005).

[19] B. A. Burrington, V. S. Kaplunovsky, and J. Sonnenschein, J. High Energy Phys. 02 (2008) 001.

[20] A. Dhar and P. Nag, Phys. Rev. D 78, 066021 (2008).

[21] E.-M. Ilgenfritz, M. Kalinowski, M. Muller-Preussker, B. Petersson, and A. Schreiber, Phys. Rev. D 85, 114504 (2012).

[22] C. V. Johnson and A. Kundu, J. High Energy Phys. 12 (2008) 053.

[23] O. Aharony, J. Sonnenschein, and S. Yankielowicz, Ann. Phys. (N.Y.) 322, 1420 (2007).

[24] G. Mandal and T. Morita, J. High Energy Phys. 09 (2011) 073.

[25] N. Callebaut, D. Dudal, and H. Verschelde, J. High Energy Phys. 03 (2013) 033.

[26] G. S. Bali and K. Schilling, Phys. Rev. D 47, 661 (1993); R. Sommer, Nucl. Phys. B411, 839 (1994).
[27] F. Preis, A. Rebhan, and A. Schmitt, J. High Energy Phys. 03 (2011) 033; arXiv:1208.0536.

[28] E. Antonyan, J. A. Harvey, S. Jensen, and D. Kutasov, arXiv:hep-th/0604017.

[29] A. A. Tseytlin, Nucl. Phys. B501, 41 (1997); R. C. Myers, J. High Energy Phys. 12 (1999) 022.

[30] K. Peeters, J. Sonnenschein, and M. Zamaklar, Phys. Rev. D 74, 106008 (2006).

[31] P. V. Buividovich, M. N. Chernodub, E. V. Luschevskaya, and M. I. Polikarpov, Phys. Lett. B 682, 484 (2010).

[32] V. V. Braguta, P. V. Buividovich, T. Kalaydzhyan, S. V. Kuznetsov, and M. I. Polikarpov, Phys. At. Nucl. 75, 488 (2012).

[33] M. N. Chernodub, Phys. Rev. D 82, 085011 (2010); Phys. Rev. Lett. 106, 142003 (2011); M. N. Chernodub, J. Van Doorsselaere, and H. Verschelde, Phys. Rev. D 85, 045002 (2012); V. Braguta, P. V. Buividovich, M. N. Chernodub, and M. I. Polikarpov, Phys. Lett. B 718, 667 (2012).

[34] V. G. Bornyakov, R. Horsley, S. M. Morozov, Y. Nakamura, M. I. Polikarpov, P.E.L. Rakow, G. Schierholz, and T. Suzuki, Phys. Rev. D 82, 014504 (2010).

[35] S. P. Klevansky, Rev. Mod. Phys. 64, 649 (1992).

[36] S. Kuperstein and J. Sonnenschein, J. High Energy Phys. 09 (2008) 012.

[37] M.S. Alam, V.S. Kaplunovsky, and A. Kundu, J. High Energy Phys. 04 (2012) 111.

[38] M. Ihl, A. Kundu, and S. Kundu, J. High Energy Phys. 12 (2012) 070.

[39] M. Ammon, J. Erdmenger, P. Kerner, and M. Strydom, Phys. Lett. B 706, 94 (2011); Y.-Y. Bu, J. Erdmenger, J. P. Shock, and M. Strydom, J. High Energy Phys. 03 (2013) 165. 